\title{
Comparison between different techniques for treatment of sub macular haemorrhage due to Age Related Macular Degeneration
}

\author{
Ahmed Abdelwahab Saad ( $\sim$ ahmed.saad@nhs.net ) \\ Daniela Vaideanu-Collins \\ James Cook University Hospital \\ Lyudmila Kishikova \\ South Tees Hospitals NHS Foundation Trust \\ Marco Isac \\ South Tees Hospitals NHS Foundation Trust \\ Dina Hamada \\ Zagazig University \\ Wael M. El-Haig \\ Zagazig University
}

James Cook University Hospital https://orcid.org/0000-0002-5159-8001

Research article

Keywords: Macular degeneration, Submacular Haemorrhage, Tissue Plasminogen activator, Vitrectomy, Subretinal injection

Posted Date: September 8th, 2019

DOI: https://doi.org/10.21203/rs.2.14176/v1

License: (9) This work is licensed under a Creative Commons Attribution 4.0 International License.

Read Full License 


\section{Abstract}

Background To compare the outcome of vitrectomy, subretinal tissue plasminogen activator (TPA), and gas with and without subretinal air versus Intravitreal TPA and gas in the treatment of sub macular haemorrhage $(\mathrm{SMH})$ due to Neovascular age related macular degeneration

Methods We analysed the notes of 29 cases presented with SMH in the period between 01/2016 and 09/2018 in James Cook University Hospital. Presenting visual acuity (BCVA), size and location of SMH, Procedure done, final BCVA at 6 months and any surgical complications were recorded. 11 Cases (Group 1) received intravitreal TPA ( $50 \mathrm{ug}$ in $0.1 \mathrm{ML}$ ), $0.3 \mathrm{ml}$ of pure SF6. 18 cases (Group 2) received $23 \mathrm{G}$ Pars Plana vitrectomy, Subretinal TPA injection (25ug in $0.1 \mathrm{ml}$ ), and 20\% SF6 gas filling. Group 2 was further divided into 2A (10 patients) who received only subretinal TPA and group 2B (8 patients) who received additional $0.1 \mathrm{ml}$ subretinal air.

Results The mean BCVA at presentation was 0.0068 in group 1 and 0.0067 in group $2(p=0.8734)$. The mean postoperative BCVA at six months was 0.31 in group 1 and 0.58 in group $2(p=0.0015)$. Subgroup analysis of group 2 didn't show statistically significant difference in outcome when adding subretinal air to the vitrectomy procedure $(p=0.7009)$.

Conclusion Vitrectomy, gas and subretinal TPA has more successful displacement rate and better visual outcome than Intravitreal TPA \& Gas alone in treating SMH involving the fovea in AMD. Additional subretinal air doesn't seem to improve the outcome in cases having vitrectcomy.

\section{Background}

Age related macular degeneration is the leading cause of blindness in the western world ${ }^{1}$. Rarely it may present with large subretinal haemorrhage involving the fovea $(\mathrm{SMH})^{2}$. Sub foveal large haemorrhages carries poor prognosis ${ }^{3}$. A few surgical techniques were described to treat $\mathrm{SMH}^{4,5,6,7,8,9,10,11,12,13}$. In this study we analysed the outcome of two commonly used methods; Intravitreal gas and TPA versus PPV, Subretinal TPA and gas. Recently some authors reported using additional subretinal air injection (subretinal pneumatic displacement) could be useful to ensure SMH displacement ${ }^{12,13}$. This study will analyse if additional subretinal air improves the outcome of patients undergoing vitrectomy.

\section{Methods}

A retrospective analysis of the notes of all patients presenting to a UK tertiary hospital with large sub macular haemorrhages due to neovascular AMD between 01/2016 and 09/2018. SMH due to trauma or choroidal polyps were excluded. On presentation all cases are typically fully assessed by full history and ophthalmic examination including OCT scan and fundus photos. The haemorrhage size and site are noted and recorded. All cases who presented within 2 weeks of the bleeding were offered treatment. The standard treatment in the unit is 23 pars plana vitrectomy, Subretinal injection of TPA ( $25 \mathrm{ug}$ in $0.1 \mathrm{ml}$ ) 
using $41 \mathrm{G}$ flexible subretinal cannula guided by the highest point in OCT (supplementary video 1) then filling the eye with $20 \%$ SF6. Additional subretinal air was done in some patients (Supplementary video 2). Some patients decline having full vitrectomy, so they were offered Intravitreal injection of TPA (50 ug in $0.1 \mathrm{ml}$ ), 0.3 of pure SF6. Avastin was used as the presenting BCVA was poor (NICE guidelines recommend Lucentis or Eylea only if BCVA is more than 4/60 (0.066). Follow up after 4 weeks with an OCT scan. If vision improves and haemorrhage is displaced, a loading dose of 3 Lucentis 4 weeks apart is prescribed. All patients were reviewed 4 weeklies with full assessment and OCT scan. The final BCVA at 6 months is recorded and compared.

\section{Results}

29 patient case notes were analysed. The age in group 1 ranged between 71 and 91 with a mean of $78+/-6$. The age in group 2 ranged between 65 and 90 with a mean of $78+/-6$ (Figure 1). Visual acuity at presentation ranged between hand movement $(\mathrm{HM})(0.005)$ and counting fingers (CF) (0.01). The mean BCVA at presentation was $0.0068+/-0.0025$ in group 1 and $0.0067+/-0.0024$ in group 2 (No statistically significant difference $p=0.8734$

The size and location of bleeding was analysed and compared. Haemorrhage size was measured by disc diameter (Figure 3) while haemorrhage location was noted if Intravitreal, Intraretinal, subretinal, sub RPE or a combination of the above (Figure 4).

Successful displacement is defined as absence of bleeding under the fovea and an area within half disc diameter around it. Successful displacement of the SMH was obtained in 9 out of 11 in group 1 (82\%) and in all cases of group $2(100 \%)$. (Figure 5 and 6 ).

The mean postoperative BCVA at six months was $0.31+/-0.18$ in group 1 and $0.58+/-0.21$ in group 2. T test was used to compare the two means and group 2 (Vitrectomy group) did better with statistically significant difference $(p=0.0015)$ (Figure 7)

To find if additional subretinal air improves the outcome, subgroup analysis of group 2 was done. Successful displacement of SMH was achieved in all cases in both subgroups. Although the mean preoperative BCVA was slightly better in Group 2A (Figure 8), the mean postoperative BCVA was slightly better in group 2B (Figure 9), Neither of the differences were statistically significant $(p=0.7009)$.

A few complications were reported in both groups. Visually significant cataract was reported in 2 cases in group 1 and 1 case in group 2. Medically controlled high IOP was reported in 3 cases in group 2 and 1 case in group 1.2 cases of retinal detachment (1 in each group) were managed with vitrectomy, cryo and gas with successful reattachment.

\section{Discussion}


AMD complicated by SMH is a serious sight threatening condition due to permanent irreversible damage to photoreceptor layer with only $11 \%$ of eyes found to have best corrected visual acuitybetter than 1.0 on Log MAR chart after two years of observation in the submacular surgery trial ${ }^{1,2}$.

Hattenbach et al. emphasized the importance of early treatment in his study, which showed thateyes with $\mathrm{SMH}$ duration of less than or equal to 14 days who had early treatment had the best visual outcome while no or poor visual prognosis is expected if the first clinical presentation was delayed for 21 days or more ${ }^{3,4}$.In this study all cases who presented within 2 weeks of the bleeding were treated.

Three different possible mechanisms have been described to explain the photoreceptor damageoccurs subsequent to large SMH. Firstly, is displacement of the neuroretina from RPE with subsequent deprivation of the photoreceptors from metabolic exchanges between the retina and choriocapillaries, secondly photoreceptor damage by shearing mechanism of retracting fibrin in the blood clot and thirdly is iron toxicity 5 .

Preventing this irreversible damage has been achieved in the last few years by different surgicalinterventions techniques share the same target of displacement of the SMH away from the fovea ${ }^{6}$ either by 1 ) intravitreal injection of tPA and gas, 2) pars plana vitrectomy + subretinal tPA + gastamponade ${ }^{7}$ with or without subretinal air injection. 3) macular translocation surgeries ${ }^{8}$ including transplantation of RPE or choroidal patch ${ }^{9}$. All the previous procedures included intravitreal injection of Anti- VEGF.

Sandhu SS et al in his retrospective case series review concluded that PPV with subretinal tPA and air tamponade followed by Ranibizumab intravitreal injection has stabilized/ improved vision in nAMD patients with SMH who failed to get haemorrhage displaced in the first attempt by intravitreal injection of tPA and gas. However, he highlighted few recurrences of the SMH and suggested that it's relevant to the altered pharmacodynamics of intravitreal anti- VEGF in vitrectomised eyes and advised of doing minimal vitrectomy with posterior vitreous detachment technique in cases with vitreomacular traction on OCT prior to the surgery ${ }^{10}$.In this retrospective series, group 2 cases were offered full vitrectomy. Further follow up and analysis may be required to determine if they need more frequent AntiVegf injections than non vitrectomised cases.

In this retrospective case series study group 2 we used $20 \%$ sulphur hexafluoride as a tamponade and added subretinal air as pneumatic subretinal displacement of the SMH in the subgroup 2A. Waizel M. et al in his retrospective analysis of 85 pseudophakic patients had undergone PPV +subretinal tPA and air or gas tamponade with $64.1 \%$ complete displacement of SMH away from fovea, highlighted that patients who had air as a tamponade achieved final visual acuity better than others who had gas tamponade either SF6 or $\mathrm{C} 2 \mathrm{~F} 6$ despite of similar anatomical outcome in the two groups ${ }^{11}$. The displacement rate is $100 \%$ in our series probably due to early intervention in all cases. 
Another promising method of displacing the SMH during vitrectomy is to inject subretinal air after the injection of the subretinal tPA. This has been described in the literatures justified by the physics principle of the higher air pressure achieved in the small subretinal space compared to the pneumatic displacement in the vitreous cavity; this leads to a higher flotation force of the air mixed with the RBCs altering its buoyancy with subsequent swift displacement of the $\mathrm{SMH}^{12}$. Kadonsono $\mathrm{K}$ et al in their prospective interventional study of 13 eyes presented with massive SMH due to $\mathrm{nAMD}$, they used specially designed 47 gauge canula and a prone position of the patient overnight after surgery and achieved $100 \%$ displacement of the $\mathrm{SMH}^{13}$, While Martel and Mahmoud used different 41 gauge cannula with post-operative recommendations of patient's upright position ${ }^{12}$. In our study group 2B achieved $100 \%$ displacement of the SMH however the final VA 6 months post-operatively was slightly better than group 2A with no statistically significant difference. The number of cases in each subgroup was probably too small to elicit a statistically significant difference.

\section{Conclusion}

Vitrectomy, gas and subretinal TPA has better visual outcome than Intravitreal TPA / Gas alone in treating SMH involving the fovea in AMD. Also, vitrectomy, gas and subretinal TPA is more efficient than Intravitreal TPA and gas in displacing SMH away from the fovea. Additional subretinal pneumatic displacement (subretinal air) doesn't seem to improve the results of vitrectomy, gas \& TPA.

\section{List Of Abbreviations}

SMH: Submacular Haemorrhage

\section{TPA: Tissue Plasminogen Activator}

\section{BCVA: Best Corrected Visual acuity}

\section{$A M D$ : Age related macular degeneration}

\section{$n A M D:$ Neovascular age related macular degeneration}

\section{PPV: Pars plana vitrectomy}

\section{RPE: Retinal pigment epithelium}

\section{VEGF: Vascular endothelial growth factor}




\section{RBCs: Red blood cells}

\section{OCT: Optical coherence tomography}

\section{HM: Hand Movement}

CF: Counting fingers

VA: Visual acuity

SF6: Sulphur hexafluoride

\section{DD: Disc Diameter}

\section{Declarations}

\section{Ethics approval and consent to participate}

Not applicable as this study is a retrospective review of outcome of different techniques (Review of notes). All patients were consented to the procedures done

\section{Consent for publication}

Not applicable for a retrospective review of data. No patient identifiable data to be published.

\section{Availability of data and material}

Master table of all the detailed results was uploaded as supplementary file. 2 videos of the surgical procedures were uploaded as supplementary files. Any further data required (e.g. imaging) could be requested from the corresponding author (Ahmed.saad@nhs.net)

\section{Competing interests}

None

\section{Funding}


None

\section{Authors' contributions}

Ahmed Saad and Daniela Vaideanu-Collins did the surgeries

Marco Isac and Lydmila Kishikova collected the data and results

Dina Hamada did the statistical analysis

Ahmed Saad wrote the manuscript

Prof El-Haig suggested the research analysis and revised the manuscript

\section{Acknowledgements}

Special thanks to Imaging team in James Cook University Hospital

\section{References}

1 Bressler NM, Bressler SB, Childs AL, et al. Surgery for hemorrhagic choroidal neovascular lesions of agerelated macular degeneration: ophthalmic findings: SST report no. 13. Ophthalmology 2004;111:19932006.

2 Wong, Chee \& Carlo Yu Alegre, Jan \& San Ian Yeo, Yew \& Cheung, Chui Ming Gemmy. (2017). Submacular hemorrhage: treatment update and remaining challenges. Annals of Eye Science. 2. 3-3. 10.21037/aes.2016.12.06.

3 Hattenbach LO, Klais C, Koch FH, Gumbel HO: Intravitreous injection of tissue plasminogen activator and gas in the treatment of submacular hemorrhage under various conditions. Ophthalmology 2001; 108: 1485-1492.

4 Van zeeburg EJ, Van meurs JC. Literature review of recombinant tissue plasminogen activator used for recent-onset submacular hemorrhage displacement in age-related macular degeneration. Ophthalmologica. 2013;229(1):1-14.

5 Toth CA, Morse LS, Hjelmeland LM, Landers MB III. Fibrin directs early retinal damage after experimental subretinal hemorrhage. Arch Ophthalmol 1991;109:723-9.

6 Stanescu-segall D, Balta F, Jackson TL. Submacular hemorrhage in neovascular age-related macular degeneration: A synthesis of the literature. Surv Ophthalmol. 2016;61(1):18-32.

7 Heriot WJ. Pre American Academy of Ophthalmology meeting, Chicago, IL, 1996. 
8 Machemer R, Steinhorst UH. Retinal separation, retinotomy, and macular relocation: li. A surgical approach for agerelated macular degeneration? Graefes Arch Clin Exp Ophthalmol. 1993;231:635e41

9 Peyman GA, Blinder KJ, Paris CL, et al. A technique for retinal pigment epithelium transplantation for age-related macular degeneration secondary to extensive subfoveal scarring. Ophthalmic Surg.

$1991 ; 22: 102 e 8$

10 Sandhu SS, Manvikar S, Steel DH. Displacement of submacular hemorrhage associated with agerelated macular degeneration using vitrectomy and submacular IPA injection followed by intravitreal ranibizumab. Clin Ophthalmol. 2010;4:637-42.

11 Waizel M, Todorova MG, Rickmann A, Blanke BR, Szurman P. Efficacy of Vitrectomy LegendCombined with Subretinal rtPA Injection with Gas or Air Tamponade. Klin Monbl Augenheilkd. 2017;234(4):487-492.

12 Martel JN, Mahmoud TH. Subretinal pneumatic displacement of subretinal hemorrhage. JAMA Ophthalmol. 2013;131(12):1632-5.

13 Kadonosono K, Arakawa A, Yamane S, et al. Displacement of submacular hemorrhages in age-related macular degeneration with subretinal tissue plasminogen activator and air. Ophthalmology. 2015;122(1):123-8.

\section{Figures}



Figure 1 
Age of patients at presentations.

\section{Mean BCVA at presentation (Decimal)}



Figure 2

Comparison between Mean BCVA at presentation (Group 1 versus Group 2).

\section{Size of vitreous haemorrhage (DD)}

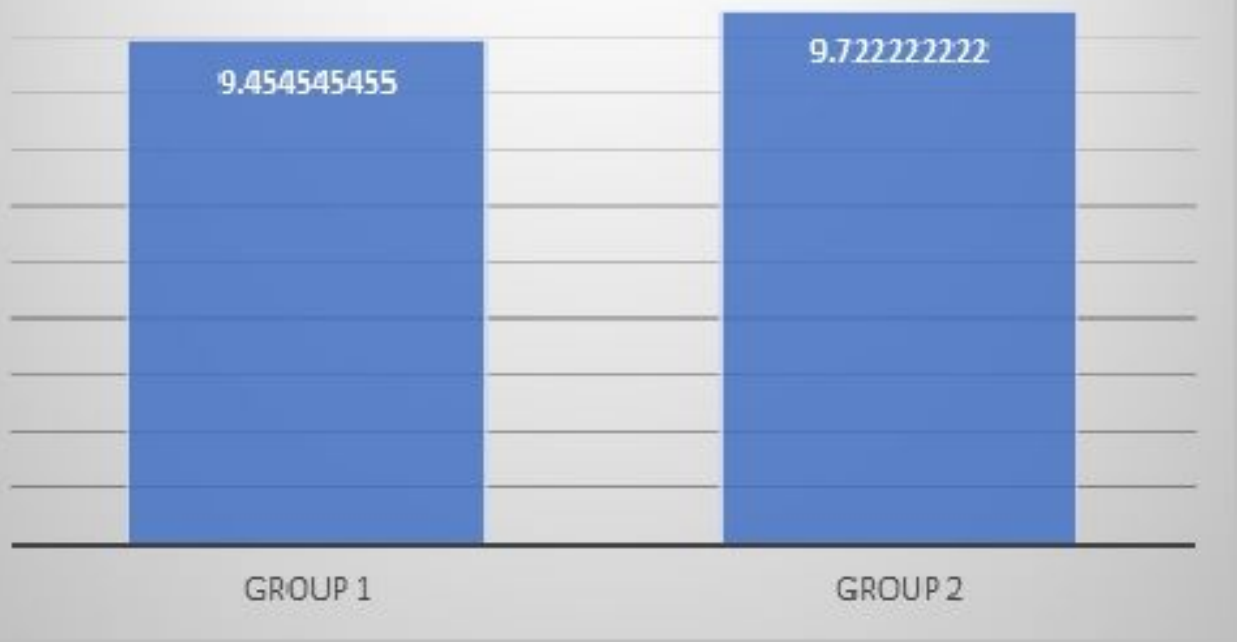

Figure 3

Size of Haemorrhage at presentation (Disc diameter) (DD). 


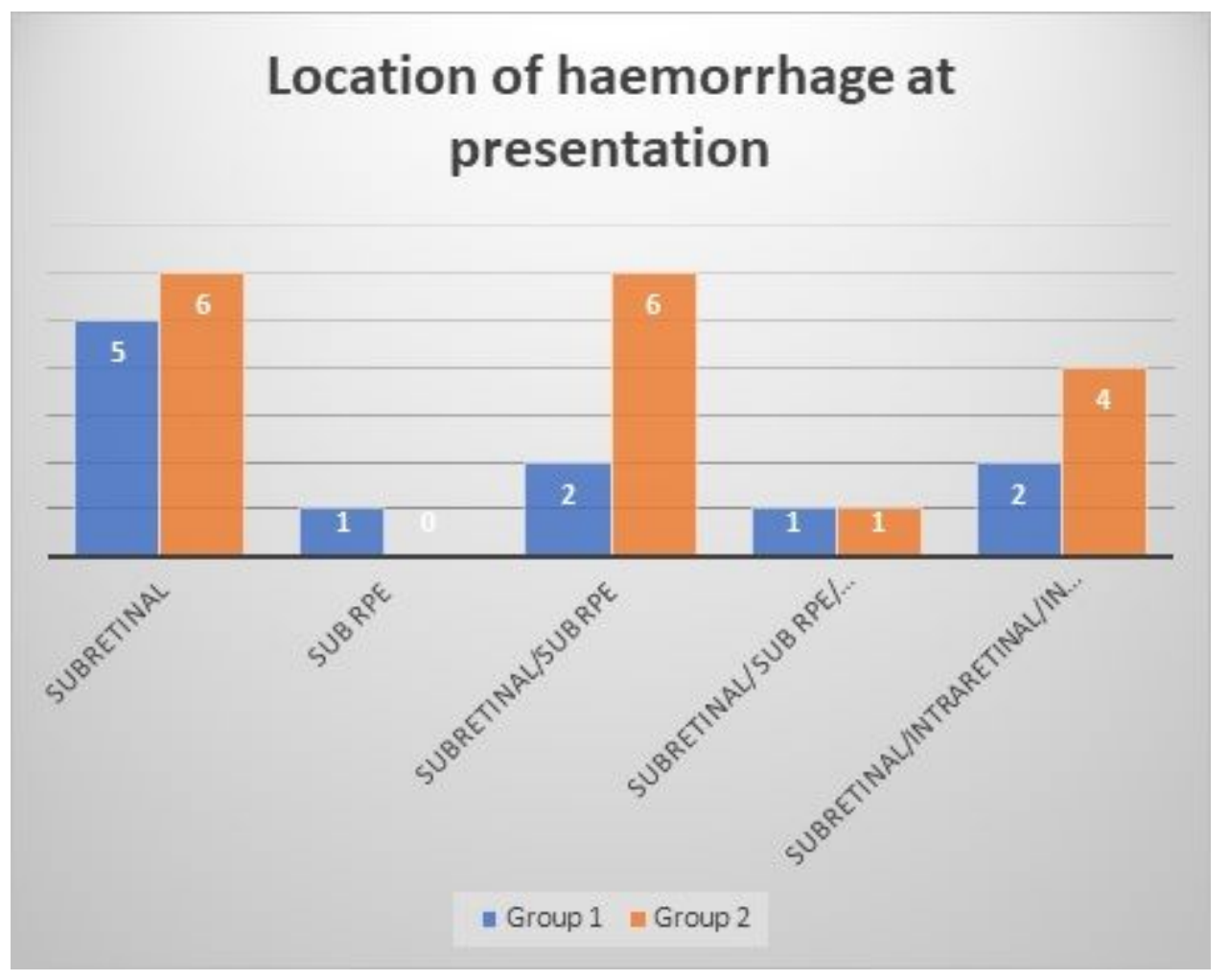

Figure 4

location of haemorrhage at presentation.

\section{Successful displacement of SMH}

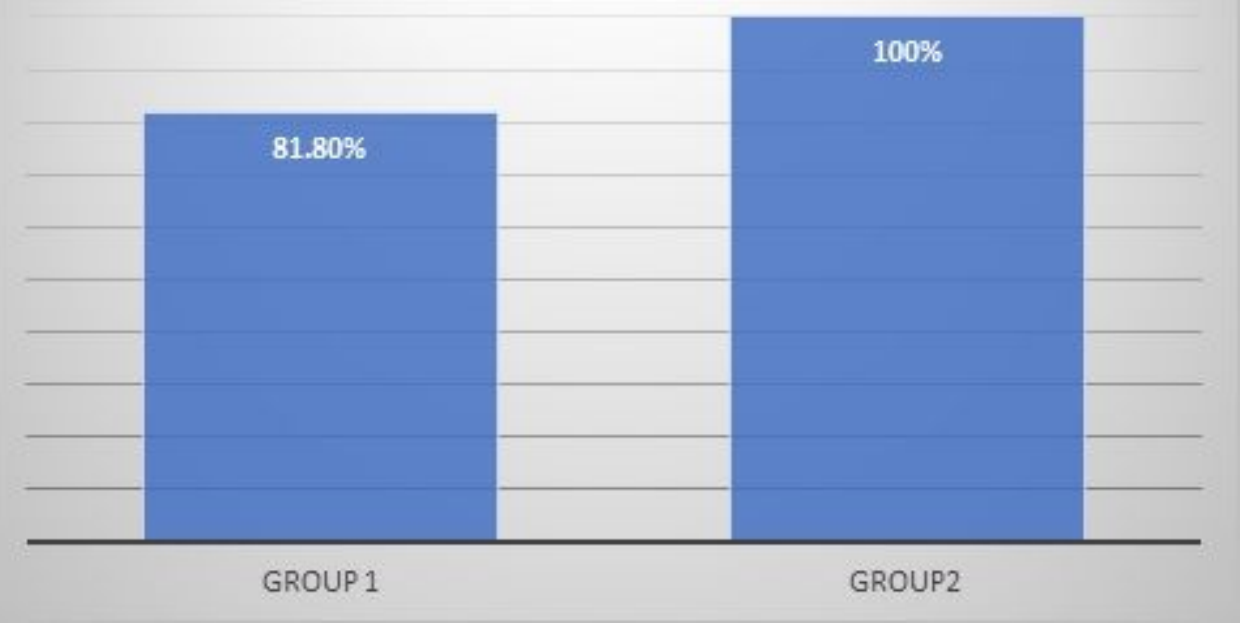

Figure 5

Successful displacement of SMH. 


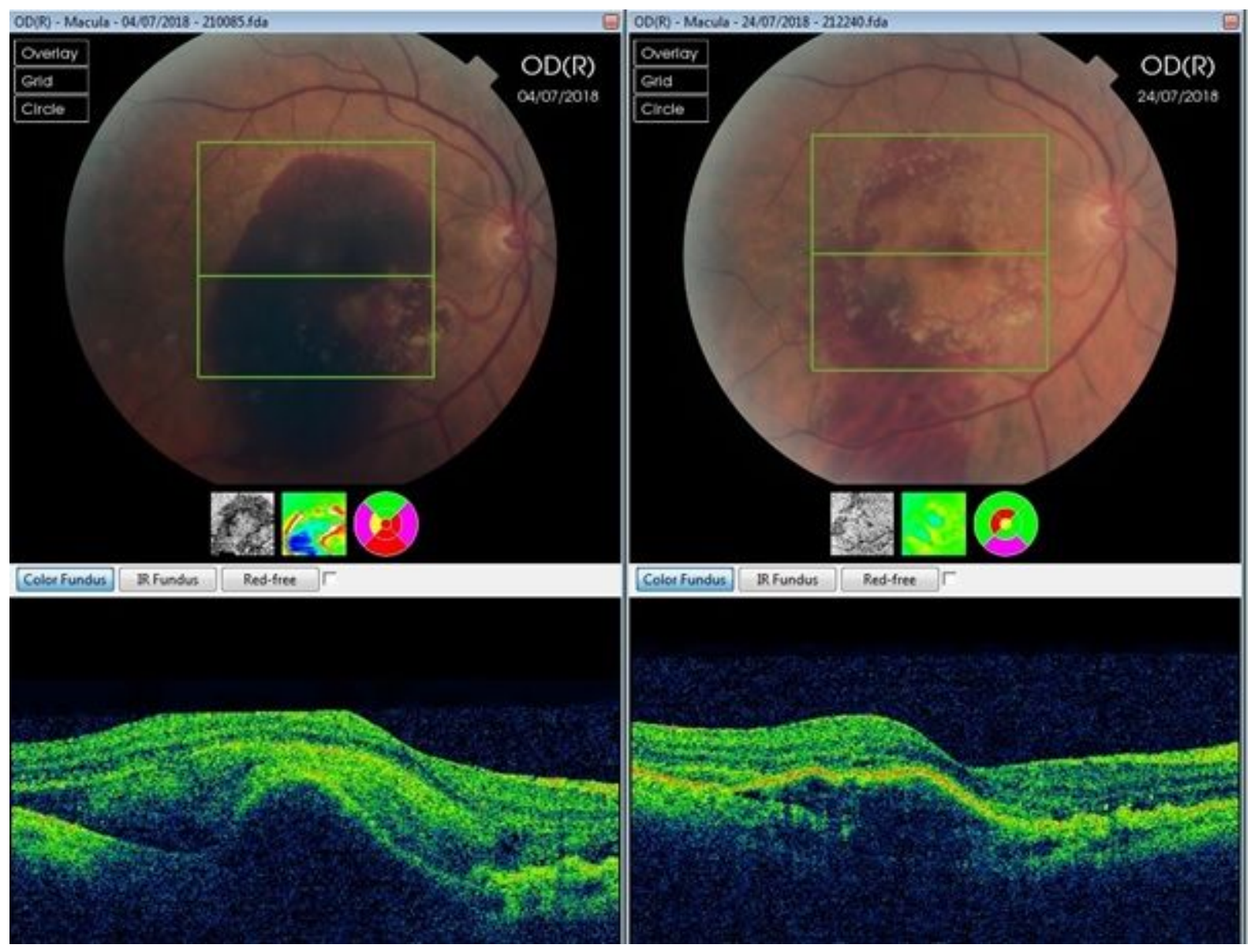

Figure 6

OCT of large Sub macular Haemorrhage due to AMD (Before and after Vitrectomy / Subretinal TPA and gas).

\section{Post Operative BCVA (Decimal)}



Figure 7

Comparison between Mean Post op BCVA in group 1 and 2. 


\section{Comparison between Mean BCVA at presentation}

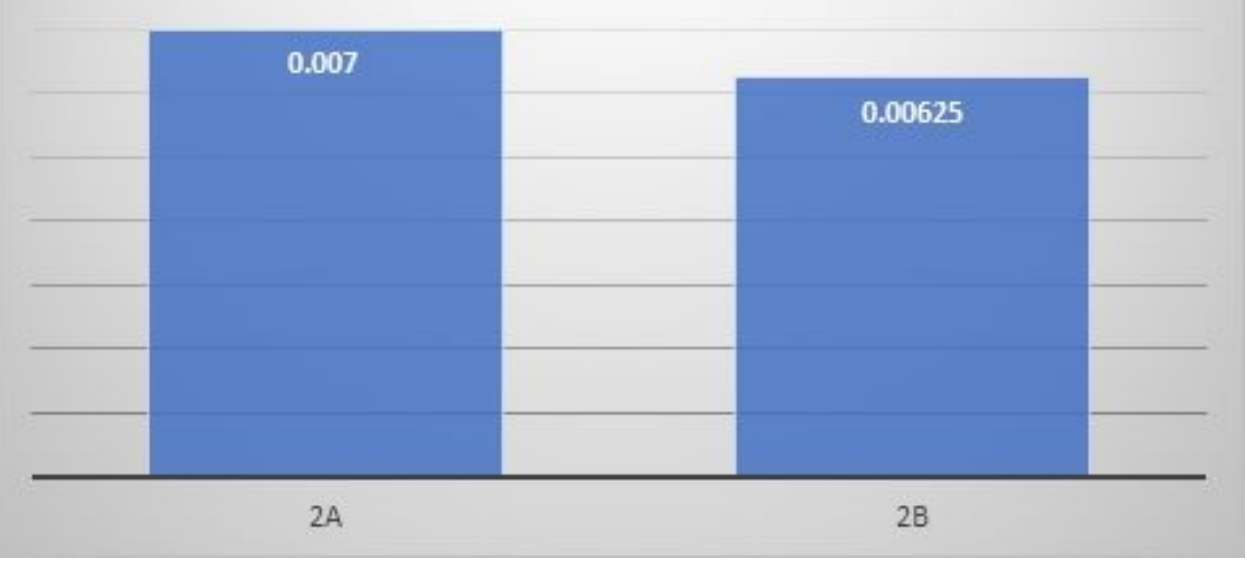

Figure 8

Comparison between Mean BCVA at presentation (Subgroup 2a versus subgroup 2b).

\section{Comparison between Mean BCVA post operatively}

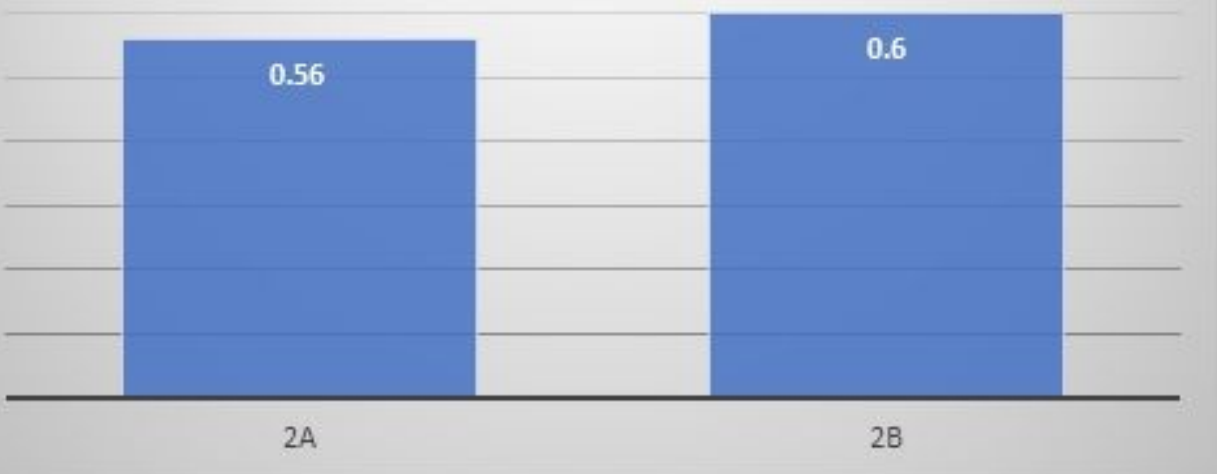

Figure 9

Comparison between Mean Post op BCVA in Subgroup group 2A and 2B

\section{Supplementary Files}

This is a list of supplementary files associated with this preprint. Click to download.

- video1.mp4

- Video2.mp4

- Resultstable.docx 